\title{
Shaping the Breast in Aesthetic and Reconstructive Breast Surgery: An Easy Three-Step Principle. Part II-Breast Reconstruction after Total Mastectomy
}

\author{
Phillip N. Blondeel, M.D., \\ Ph.D. \\ John Hijjawi, M.D. \\ Herman Depypere, M.D., \\ Ph.D. \\ Nathalie Roche, M.D. \\ Koenraad Van Landuyt, \\ M.D., Ph.D. \\ Ghent, Belgium
}

This is Part II of four parts describing the three-step principle being applied in reconstructive and aesthetic breast surgery. Part I explains how to analyze a problematic breast by understanding the main anatomical features of a breast and how they interact: the footprint, the conus of the breast, and the skin envelope. This part describes how one can optimize results with breast reconstructions after complete mastectomy. For both primary and secondary reconstructions, the authors explain how to analyze the mastectomized breast and the deformed chest wall, before giving step-by-step guidelines for rebuilding the entire breast with either autologous tissue or implants. The differences in shaping unilateral or bilateral breast reconstructions with autologous tissue are clarified. Regardless of timing or method of reconstruction, it is shown that by breaking down the surgical strategy into three easy (anatomical) steps, the reconstructive surgeon will be able to provide more aesthetically pleasing and reproducible results. Throughout these four parts, the three-step principle will be the red line on which to fall back to define the problem and to propose a solution. (Plast. Reconstr. Surg. 123: 794, 2009.)

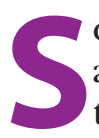

culpting a flap of autologous tissue into an aesthetically pleasing breast has become the most demanding aspect of breast reconstruction, as the technical aspects of flap harvesting and microsurgery have become routine in most centers. ${ }^{1-9}$ There is no question that breast reconstruction is an important component in the final recovery of many breast cancer patients and is a main contributor to the quality of life of the post-breast cancer patient. An unreconstructed mastectomy defect but also a poorly executed reconstruction serves as a constant reminder of a cancer diagnosis. Therefore, it stands to reason that the more aesthetic and natural a reconstructed breast appears and feels, the more completely a breast cancer patient will recover. Indeed, in an era where the success rates of elective microvascular breast reconstruction approach 100 percent, a flap that "survives" but fails to recreate

From the Department of Plastic and Reconstructive Surgery and the Division of Gynaecological Oncology, University Hospital Ghent.

Received for publication April 27, 2008; accepted August 20, 2008.

Copyright (C2009 by the American Society of Plastic Surgeons

DOI: 10.1097/PRS.0b013e318199ef16 an aesthetic breast is appropriately judged a reconstructive failure.

Why then does so little literature exist on this critical and challenging aspect of breast reconstruction? Three-dimensional thinking and the ability to manipulate tissue in three dimensions are skills that typically need to be well developed by plastic surgeons. Despite these talents, the mere fact of adequately describing the manipulation of an essentially flat structure such as a skin and fat flap into a three-dimensional structure such as a breast is challenging and difficult, particularly in writing.

Recreating an aesthetically pleasing breast after partial or total mastectomy is a combination of good measurements and artistic insight. The experience of the surgeon is the final determining factor. Unfortunately, artistic insight and experience are very abstract and ill-defined elements. In this article, we will put forward a very systematic

Disclosure: None of the authors has a financial interest to declare in relation to the content of this article. 
approach for recreating the female breast after radical, modified radical, and skin-sparing mastectomies and giving the new breast attractive dimensions and proportions.

This consists of three important steps: creating the breast footprint on the chest wall, placing a proper conus on the footprint, and redraping the appropriate skin envelope over the conus. If each step is executed properly, the combination of these three steps will create an aesthetically pleasing breast even in the most difficult reconstructions. The breakdown of the reconstructive process into three basic steps makes it easier for the surgeon to understand and analyze the problem and will facilitate a step-by-step buildup of the breast.

\section{PREOPERATIVE STRATEGY FOR GOOD RESULTS}

Patient selection is always very important. This article specifically describes not only how to rebuild an entire breast after ablative surgery but also the reconstruction of a subtotal absence of formation of the breast after congenital agenesis or extreme hypotrophy. Extreme cases of Poland syndrome can be reconstructed following the same principles. The patient's desire to undergo such a reconstruction is probably the most important factor in patient selection. Different techniques of implant and autologous tissue reconstruction need to be explained to the patient, together with all of the possible complications and disadvantages. We avoid implant reconstruction in patients who have undergone irradiation. We also feel that we can provide a more pleasing aesthetic result with autologous tissue compared with implants.

Potential donor sites are evaluated to determine which site will be used, taking into consideration the condition of the receptor site and the patient and surgeon preference. If sufficient tissue is present at the lower abdominal wall, the transverse rectus abdominis musculocutaneous (TRAM) ${ }^{1,2}$ or deep inferior epigastric artery perforator flap ${ }^{4-7}$ will provide sufficient adipose tissue bulk and more than sufficient skin. The superior or inferior gluteal artery perforator flap ${ }^{8,9}$ is always bulky, but the skin paddle is relatively small. For that reason, gluteal artery perforator flaps offer the best results in primary reconstructions where most of the skin envelope is preserved. Gluteal artery perforator flaps generally have a more rigid consistency, making shaping slightly harder to perform. Therefore, gluteal artery perforator flaps are chosen only if the lower abdominal wall cannot be harvested. Latissimus dorsi flaps ${ }^{3}$ or thoracodorsal artery perforator flaps allow the harvest of large skin islands but generally the total volume of the flap is not large enough to reconstruct the breast completely, necessitating the addition of an implant. Thoracodorsal artery perforator flaps are the first choice in partial breast reconstruction of the lateral quadrants of the breast (see Part III).

If an implant reconstruction is preferred, the appropriate shape, size, and volume need to be selected. If an important part of the skin envelope is missing, a temporary expansion of the remaining skin needs to be performed to acquire sufficient ptosis in the new breast. All this demonstrates that the choice of reconstructive tool or type of flap will directly influence the final aesthetic outcome. A wrong choice of flap may lead to a situation where a good result may never be obtained regardless of all the secondary corrections that would be performed afterward.

The degree of surgical postablative and radiotherapy damage to the three different anatomical structures at the recipient site also has an influence on the final result. More aggressive ablative surgery, higher doses of radiotherapy (or higher sensitivity to radiotherapy), the number and type of previous reconstructive attempts, and the absence of the nipple-areola complex will all make the reconstructive procedure more complex and negatively influence the final result. It will be easier to recreate the normal shape of a breast after an immediate skin-sparing mastectomy where the borders of the footprint have been respected compared with the secondary reconstruction after radical mastectomy intensively damaged by radiotherapy where the footprint, conus, and envelope have been damaged dramatically. The preoperative analysis of the different problems at the three anatomical levels can be clearly marked on the chest wall with detailed preoperative drawings. These markings are explained below for the different situations encountered after mastectomy. In the following sections, we describe a step-bystep approach to autologous and implant breast reconstruction after mastectomy by breaking up the analysis of the problem and the surgical solution for the footprint, the conus, and the skin envelope.

\section{AUTOLOGOUS BREAST RECONSTRUCTION}

\section{Delayed Unilateral Autologous Reconstruction}

\section{Footprint}

In a secondary breast reconstruction, the patient presents with a breast gland that has partially or totally been removed and with overlying skin of 
the breast that is partially or totally preserved. The skin may be irradiated or scarred or damaged by previous infections, hematomas, or other means. In modified radical mastectomy cases and all cases where the inframammary fold can no longer be identified, it is important to determine the level of the new inframammary fold. Mostly, this is positioned approximately 2 to $3 \mathrm{~cm}$ higher than the contralateral inframammary fold (Fig. 1). If significant skin fibrosis and contraction are present at the mastectomy site, the fold should be positioned slightly higher. Simple incision and resection of that scar will release all surrounding tissue and the skin will shift caudally. Soft pliable tissue will not move as much and therefore the inframammary fold can be placed slightly lower. By pulling on the abdomen later, this fold will move down even farther and the normal inframammary fold will stay in place. When in doubt, it is always better to put the new inframammary fold a little higher than too low. Correcting an inframammary fold that is positioned too high is much easier to perform than raising the fold in a secondary procedure.

Surgery starts by excising all scars and severely scarred tissue that lie over the footprint. In modified radical mastectomy cases, the new inframam-

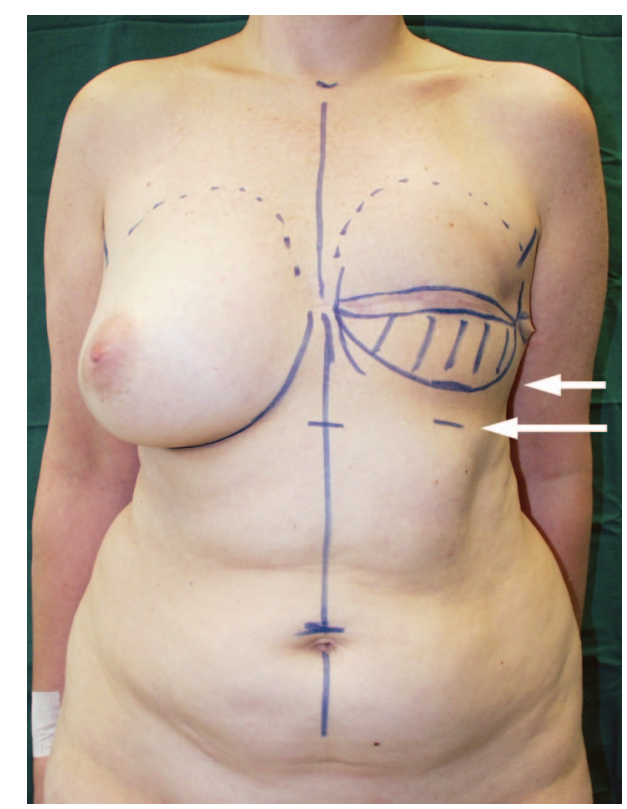

Fig. 1. The footprint of the new breast can be estimated from the contralateral breast. Upper, medial, and lateral borders are marked, as is the mastectomy scar. The new inframammary fold (short arrow) is positioned 2 to $3 \mathrm{~cm}$ higher than the existing contralateral inframammary fold (lower longer arrow). The striped area between the new inframammary fold and the mastectomy is deepithelialized. mary fold is incised following the lines marking the limits of the new breast footprint (Fig. 1). The incision is performed to a depth of approximately $1 \mathrm{~cm}$ to allow easy suturing of the flap to the skin edges of the inframammary fold. The skin in between the mastectomy scar and the inframammary fold is only deepithelialized. In this way, we preserve a layer of 1 to $2 \mathrm{~cm}$ of fat in the lower parts of the breast that will assist in improving projection of the lower pole. The skin edges of the upper mastectomy flap are thinned down to the dermis for the first $5 \mathrm{~mm}$ and then progressively trimmed to obtain a fluid and stepless transition of the mastectomy flap into the upper skin edge of the flap. The upper mastectomy flap is then further undermined up to the preoperative markings on the lateral, cranial, and medial borders of the breast footprint. The internal mammary artery and vein are subsequently prepared for anastomosis.

\section{Conus}

The conus is made up by the bulk of a lower abdominal or gluteal flap. The principle of shaping the lower abdominal flap applies to all myocutaneous flaps (pedicled, free, or muscle-sparing TRAM) or perforator flaps (deep inferior epigastric artery perforator). The same goes for myocutaneous or perforator flaps from the upper or lower gluteal area (superior or inferior gluteal artery perforator). The art consists of transferring a certain volume to the recipient site and turning a flat fat structure into a three-dimensional asymmetrical conus.

In a unilateral reconstruction, we harvest both halves of the lower abdomen centered over the entire (pedicled TRAM) or part of (free musclesparing TRAM) the rectus abdominis muscle or the most dominant perforator (deep inferior epigastric artery perforator flap) as a single unit, allowing maximum flexibility in breast shaping once the flap is revascularized. It is rare that a breast is so large and the abdomen so thin that the entire lower abdomen needs to be used to obtain adequate volume. If necessary in free flap surgery, the pedicles can be harvested on both sides and anastomosed to each other or to two different sets of recipient vessels. We discourage the use of bipedicled TRAM flaps because of their important donor-site morbidity and limited freedom for shaping the flap. We routinely explore both sides of the abdomen in an effort to find the best available perforators. There is a preference nevertheless to use the vessels of the contralateral hemiabdomen. Using these vessels and turning the flap 180 degrees allows for easy microsurgery, eventual sensory nerve suturing to the lateral intercostal 
nerve(s), and easy access to the superficial inferior epigastric vein when it is necessary to improve venous outflow. Turning the flap 180 degrees also places the bulk of the thicker midabdominal region in the inferior pole of the breast, contributing to the asymmetric projection of the breast conus.

After the anastomoses, the shaping of the conus starts (Fig. 2, above, left). A wedge of skin is removed over the periumbilical region and closed in two layers. By so doing, more volume is gathered together in the lower half of the flap. The more skin that is removed, the more projection that is achieved but the more compression of the subdermal plexus in this area that should be expected (Fig. 2, above, right). Shaping starts at the pectoralis tendon.

In lower abdominal flaps with a contralateral pedicle, the contralateral tip of the flap is fixed just below the pectoralis tendon by suturing Scarpa's fascia to the pectoralis fascia 2 to $3 \mathrm{~cm}$ medial to the lateral pectoral muscle border in the superolateral edge of the footprint. In lower abdominal flaps with an ipsilateral pedicle, the most remote and poorest vascularized contralateral part of the flap (previously called zone IV) needs to be resected first after a correct estimation of volume has taken place (Fig. 2, above). Next, the contralateral corner of the remaining flap is sutured just below the pectoralis tendon in the same fashion (Fig. 2, second row, left). This first key suture and filling of the upper lateral part will recreate the anterior axillary fold. In the next steps, skin staples are used to create a temporary shape of the conus. Once a final shape is achieved, the staples are replaced by permanent sutures.

The lateral edge of the flap is then stapled to the most lateral part of the inframammary fold under slight tension. Sufficient tension needs to be applied nevertheless to avoid excessive lateral fullness. The transition from the lateral pectoral border into the lateral portion of the flap also recreates a natural lazy-S shape along the lateral border of the breast (Fig. 1). This lazy-S contour should also be a smooth transition into the tissues of the lateral thorax. The exact position of the lateral key suture is determined by shifting the flap along the inframammary fold while assessing the lazy-S contour. By shifting the flap laterally, more lateral fullness will be obtained (Fig. 2, second row, right). Pulling the lateral edge of the flap more medial will flatten the lateral part of the new breast (Fig. 2, third row, left). It is always better to have minimal fullness at the time of reconstruction, as the flap will shift laterally and distally in the postoperative period.
Medial to the second key suture and around the midclavicular line, the skin of the flap is bunched up. This effect together with the triangular skin resection around the umbilicus will dramatically increase the projection of the flap (Fig. 2, third row, right). This will also help in creating a straight angle between the skin of the flap and the abdominal skin below the inframammary fold.

The third key suture is placed at the medial end of the inframammary fold where it extends most medially. In the medial part of the inframammary fold, the flap is no longer bunched together to avoid overfilling of the inferomedial quadrant of the new breast. In contrast, if the flap is not placed medially enough, it can be difficult to achieve sufficient medial cleavage.

At this point, in lower abdominal flaps with a contralateral pedicle, two important points need to be considered. First, a volume estimation must be performed in cases where the surgeon attempts to obtain a volume comparable to the contralateral side. Second, the most remote and most poorly vascularized contralateral part of the flap (previously called zone IV) needs to be evaluated for its vascular supply. This can be accomplished by visual inspection of the skin or by carving into the dermal plexus with a Bistouri in the most distal corner. All poorly vascularized tissue needs to be resected immediately. Tissues that show mixed arterial and venous bleeding can be kept. More tissue from this region can obviously be resected if the contralateral breast is smaller.

We generally make the reconstructed breast 5 to 10 percent larger than the contralateral one, anticipating some decreased swelling postoperatively. If the contralateral breast is hypertrophic and the patient has expressed the desire to have a reduction on that side, a breast is constructed with well-vascularized tissue that fits with the proportions of her torso. In thinner patients, the final volume will depend on whatever fat volume is present on the lower abdominal wall. If the patient wishes to enlarge the contralateral breast, a larger flap is taken, if available, and a larger breast is constructed.

In lower abdominal flaps with an ipsilateral pedicle, the same decision making with regard to volume has occurred earlier. The inner, ipsilateral tip of the flap is best rounded off by resecting a few centimeters of skin. This will provide a nice medial contour with a smooth transition into the presternal region (Fig. 2, third row, right). The subcutaneous fat at the border of the upper mastectomy flap sometimes needs to be trimmed to allow for a gradual transition of the flap skin into the upper mastectomy skin flap. 

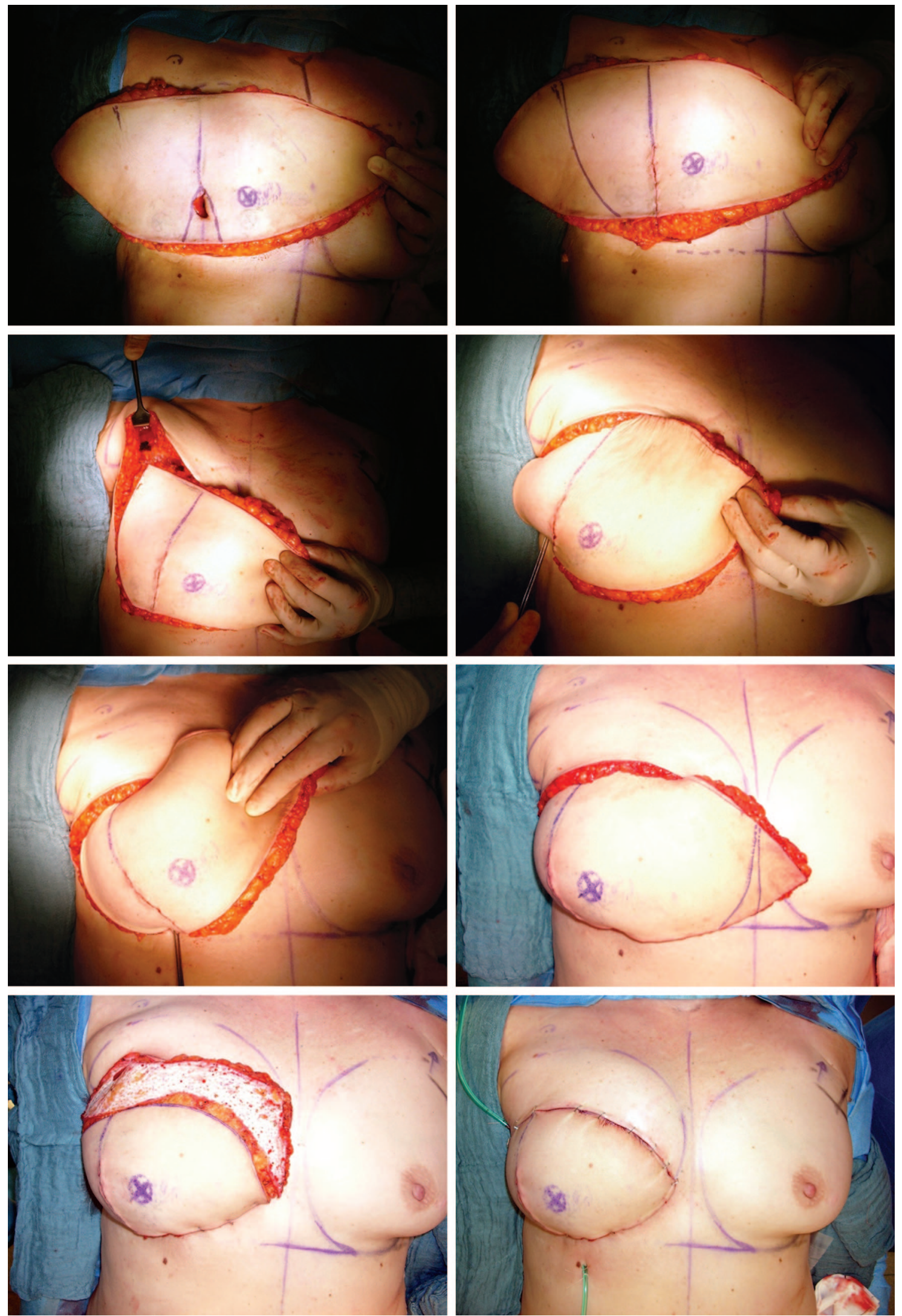

Fig. 2. Photographs showing step-by-step shaping of a deep inferior epigastric artery perforator flap in a secondary autologous breast reconstruction. (Above, left) The flap is positioned with an ipsilateral perforator (blue X) over the 
Finally, it is important to slightly overfill the superomedial quadrant of the breast with the flap. Sometimes, the superior part of the flap may need to be sutured to the chest wall to pull up the conus in the region of the infraclavicular fill. Overfilling this region will correct itself, as gravity will be pulling the flap caudally over the ensuing 6 months. It is also better to have too much fat in this region than too little. Excessive fat can easily be removed later by liposuction, whereas a depression in this area is very disturbing for the patient, as it is often exposed in the décolleté area. Also, complex procedures are often necessary in a later phase to add fat to that area. Finally, the upper part of the flap is deepithelialized according to the dimensions of the upper mastectomy flap (Fig. 2, below).

Creating a nice conus out of a gluteal flap can be much more challenging. Overall, the gluteal flap is shorter (24 to $34 \mathrm{~cm}$ ) and of limited width (maximum, $12 \mathrm{~cm}$ ). The flap is overall thicker but, more importantly, much more consistent and firm than the abdominal flap. Before starting the inset of the flap, both tips of the flap are thinned by obliquely resecting fat below the skin (Fig. 3). By so doing, the oblique angles of the ideal conus are mimicked and a natural transition into the chest wall can be provided at the superolateral and medial parts of the breast. One tip is sutured to the pectoralis tendon as described above.

Further shaping progresses in the same fashion as with an abdominal flap. After placing the second key suture at the lateral part of the inframammary fold, the gluteal flap does not need any bunching. The rigidity of the flap creates sufficient projection around the midclavicular line. The medial tip of the flap can be further trimmed to achieve a progressive transition into the presternal area. Because of the limited width of the flap, it is very typical to find at the end of the shaping procedure a shortage of tissue at the superior border of the flap. This will cause a depression or cavity that needs to be corrected by redraping the

Fig. 2. (Continued) right breast. (Above, right) The least vascularized zone of the flap has been marked for removal and a triangular excision of skin over the umbilicus has been performed. (Second row, left) The contralateral corner of the remaining flap is sutured just below the pectoralis tendon. (Second row, right and third row, left) The lateral fullness of the breast is determined: the more the flap is moved medially, the less bulging in the lower outer quadrant. (Third row, right) The medial flap excess is determined and resected. (Below, left) Deepithelialization of the upper part of the flap and the final result (below, right) are shown.

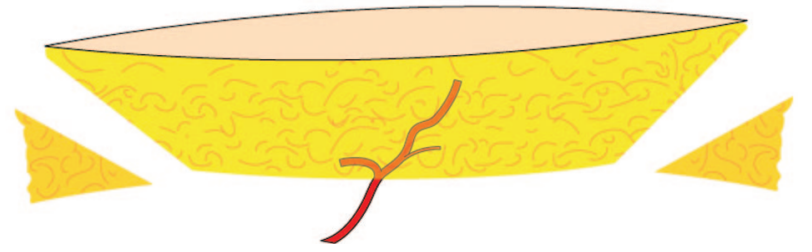

Fig. 3. Superior and inferior gluteal artery perforator flaps are more rigid and more difficult to shape. A long, narrow, thick gluteal flap needs to be thinned at its tips to ensure a smooth transition into the chest wall. Basic shaping at the first operation consists only of beveling both corners of the flap.

flap or lipofilling during the second procedure 6 months later.

\section{Envelope}

Increased skin requirements are the obvious difference between immediate and delayed breast reconstruction. Attractive breast projection and shape result from a combination of the innate projection of the conus and an appropriately tailored skin envelope. Even a perfectly shaped flap will fail to achieve an aesthetic breast if the overlying mastectomy skin is inelastic, redundant, and ill fitting. In secondary reconstructions, the skin envelope is composed of both the upper mastectomy flap and the skin of the flap itself. The level of the horizontal or oblique mastectomy scar will determine the shares of mastectomy skin and flap skin. If in any way the upper mastectomy flap is scarred, retracted, or damaged, one must consider either resecting a part or releasing the skin on the inside by transecting and scoring constricting bands. Often, in the lateral part of the skin envelope toward the axilla, excessive scarring can be found, limiting the expansion of the upper flap. Dissection and scar release down into the axilla is sometimes necessary. After mastectomy, skin has shifted toward the axilla. Also, this skin needs to be recruited and brought forward. By putting one hand underneath, the upper skin flap should be easily lifted up from the chest wall and no restricting bands should be felt. In no way should the upper mastectomy skin flap compress the lower abdominal flap.

Once the final volume of the flap is determined, one can make a rough estimation of how much skin paddle will be required. The flap can be pushed up into the upper pouch or pulled down, leaving, respectively, less or more vertical skin height on the skin paddle of the flap. The more skin that is left at the midclavicular line, the more ptosis that will be achieved. The more the skin of the flap is resected in the lateral part of the flap, the more the flap will be pushed medially. Once again, the 
clinical and aesthetic assessment of the contralateral breast becomes key in fine tuning the shape. Either the surgeon attempts to mirror the contralateral breast if the other side is not to be touched or the surgeon creates a totally new attractive shape if a mastopexy or other shape adjustment is foreseen at the contralateral breast. In the former case, exact vertical and horizontal measurements will help in obtaining a symmetrical result. The part of the skin paddle that will be covered by the upper mastectomy flap is consequently deepithelialized.

\section{Immediate Unilateral Reconstruction}

\section{Footprint}

It is crucial to identify whether the mastectomy procedure has violated the normal boundaries of the breast footprint. If so, it is very important to meticulously repair the footprint and verify its height and width on the chest wall. The preoperative markings of the borders of the breast need to checked and if necessary resutured to the chest wall. The most commonly violated borders are the inframammary fold and the lateral border of the breast, particularly after an axillary dissection. Particular attention should be given to the inframammary fold, as this will be the main support of the new breast. Whether or not the pectoral muscle is resected is not an issue with an autologous reconstruction. Meticulous preparation of the footprint will facilitate insetting of the flap and will reduce the number of sutures that need to be placed directly into the flap.

\section{Conus}

In unilateral reconstruction with any flap, the weight of the flap and of the mastectomy specimen provide guidance for gross trimming of the flap. As with delayed reconstructions, a breast will be reconstructed that will be 10 percent larger than the original breast, particularly if radiotherapy is expected. If not enough flap volume is available, a contralateral breast reduction needs to be considered or an augmentation procedure (implantation or lipofilling) performed in a second operation 6 months later.

The shaping of lower abdominal, gluteal, or other flaps used for primary (immediate) reconstruction is performed exactly the same way as with delayed reconstructions. Any type of suture instead of staples is used to suture the edge of the flap to the superficial fascia at the inframammary fold. Otherwise, a basic shape is given to the flap using the three key sutures as described above. The skin is draped gently over the flap and the shape is evaluated. The key sutures are adjusted until an acceptable shape is obtained. A few additional resorbable sutures can help redistribute the fat better and spread the fat over the entire footprint. However, we have noticed that the more sutures that are placed, the less natural the result. The volume of the flap needs to spread itself out over the footprint and provide sufficient anterior and circumferential pressure to keep the skin envelope under tension.

\section{Envelope}

The availability of an abundant skin envelope is a major difference between immediate and delayed breast reconstruction. Excessive skin can be resected if the reconstructed breast is smaller than the original breast. In excessively ptotic breasts, a mastopexy needs to be performed at the initial reconstruction using a keyhole or Wise pattern. Caution needs to be used in smokers or in patients who have had their skin flaps thinned excessively during mastectomy. In these situations, extensive skin excisions are best delayed to a secondary shaping procedure. Clearly devascularized mastectomy flaps should be débrided back to viable skin. The portion of the skin paddle that will be covered by the mastectomy flap is consequently deepithelialized.

In immediate reconstruction, once the new breast pocket, footprint, volume, and projection are established, the final tailoring of the skin envelope is determined. With the flap in position, staples are used with a tailor-tacking method to determine the optimal amount and orientation of skin excision. Skin islands of the flap can be left as patches in between the mastectomy skin to fill up areas of limited skin resection. Additional flap skin needs to be left to be able to perform the nipple reconstruction in a second operation. Drains are used to suck the skin envelope to the flap surface, thereby limiting subcutaneous collections, scarring, and shape deformities.

\section{Bilateral Reconstruction}

\section{Footprint}

The footprint for both breasts is prepared in the same way as for unilateral, immediate or delayed, reconstructions.

\section{Conus}

The main difference between unilateral and bilateral immediate breast reconstruction relates only to the availability of an adequate amount of donor tissue. If the lower abdomen is preferred, the flap is divided into two (symmetrical) halves early during flap harvest. This allows one team to proceed with microsurgical anastomosis of the first flap while the second flap is harvested. To 
increase the volume of the flap, the lateral corner of the flap design can be extended farther laterally and can be expected to completely survive because the pedicle needs to perfuse only half of the abdomen. By splitting the abdomen in two, less volume will be available. Therefore, there will be less liberty to shape the breast through the process of sculpting an excessively large flap until the desired shape is obtained. Shaping needs to be performed by repositioning and redistributing the fat on the chest wall.

After harvesting, the contralateral triangular hemiabdomen is rotated 180 degrees and placed on the footprint with the lateral tip of the flap oriented toward the pectoralis tendon (Fig. 4, above, left). The lower skin incision of the flap is now at the cranial border of the new breast and the skin around the umbilicus is pointing caudally. In this position, the thickest part of the flap is placed in the lower pole and the thinnest part is spread out in the upper lateral part of the breast. Also, the superficial inferior epigastric vein protrudes in the cranial part, allowing for easy anastomosis with the internal mammary, external jugular, or cephalic vein. As performed in unilateral reconstructions with a contralateral pedicle, the first two key sutures are placed just below the pectoralis tendon (Fig. 4, above, right and second row, left) and at the most lateral point of the inframammary fold. Instead of bunching skin together, the necessary projection is obtained by turning the periumbilical part of the flap underneath the most caudal part of the flap (Fig. 4, second row, right), thereby folding the flap under itself (Fig. 4, third row, left). The more tissue that is rotated under the flap, the more projection that can be achieved but the more cranial height of the flap that is lost. The midline inferior incision angle of the flap is placed at the medial border of the footprint. The vertical height of this angle is determined by the balance between sufficient cleavage and sufficient fullness of the upper medial quadrant of the new breast. Shifting this part of the flap up and down the medial border of the footprint will quickly show the ideal proportions in terms of shape and volume distribution. A third key suture is placed at the medial border. Skin is tightened in a vertical direction at the inframammary fold by adding a few skin staples until a nicely rounded inferior curvature of the flap is found (Fig. 4, third row, right). If the flap is too large, fat can be resected from the medial border of the flap (Fig. 4, below, left). Thinning of the subcutaneous fat at the border of the upper mastectomy skin is crucial for obtaining a progressive transition between flap and mastectomy skin (Fig. 4, below, right).

In case we need the superior gluteal artery perforator flap for bilateral reconstruction after bilateral mastectomy, we propose a two-step procedure to our patients. A single surgeon, with or without surgical assistance, often prefers to transfer only one flap at the time, with a 3- to 6-month interval, because of the prolonged operating time. Obviously, shaping the superior gluteal artery perforator flap in two different steps for a bilateral breast reconstruction is identical to a unilateral primary or secondary reconstruction.

\section{Envelope}

Once the conus is formed, the remaining mastectomy skin will cover the flap. Draping of this skin is performed in the same way as in immediate or delayed unilateral reconstructions described above.

\section{GENERAL ISSUES IN AUTOLOGOUS RECONSTRUCTION}

Although it is not the purpose of this article to expand on secondary corrections, it is obvious that the final result cannot be achieved after one operation. The goal of the first operation, regardless of which reconstructive technique or flap was applied, is to obtain a basic shape that resembles a normal breast quite closely. Frequently, a second operation is needed 6 months later to adjust the reconstructed breast, the contralateral breast, or both. Ipsilateral corrections include nipple reconstruction, scar revisions, volume adjustments either by reduction (i.e., direct resection or liposuction) or by augmentation (i.e., implants, additional flaps, or lipofilling), and shape corrections. This can be accomplished by repositioning and/or rotating the flap or implant, skin resections, and adjustments of the inframammary fold or other borders of the flap. The contralateral side may be adjusted by the common techniques of augmentation, reduction, and mastopexy. Symmetry is our final goal after the second procedure.

In reality, it is not always easy to obtain that symmetry, and in specific cases it might be necessary to repeat flap corrections and adjustments of the contralateral breast. These small "nip-andtuck" procedures are very individual, can almost always be performed under local anesthesia, and need to be repeated until the patient with realistic expectations is happy with the result. The opinion and wishes of the surgeon are important but always secondary to those of the patient.

In a third phase, 3 months after the last corrective procedure, a tattoo of the areola is performed. As our eyes are very sensitive to color differences, 

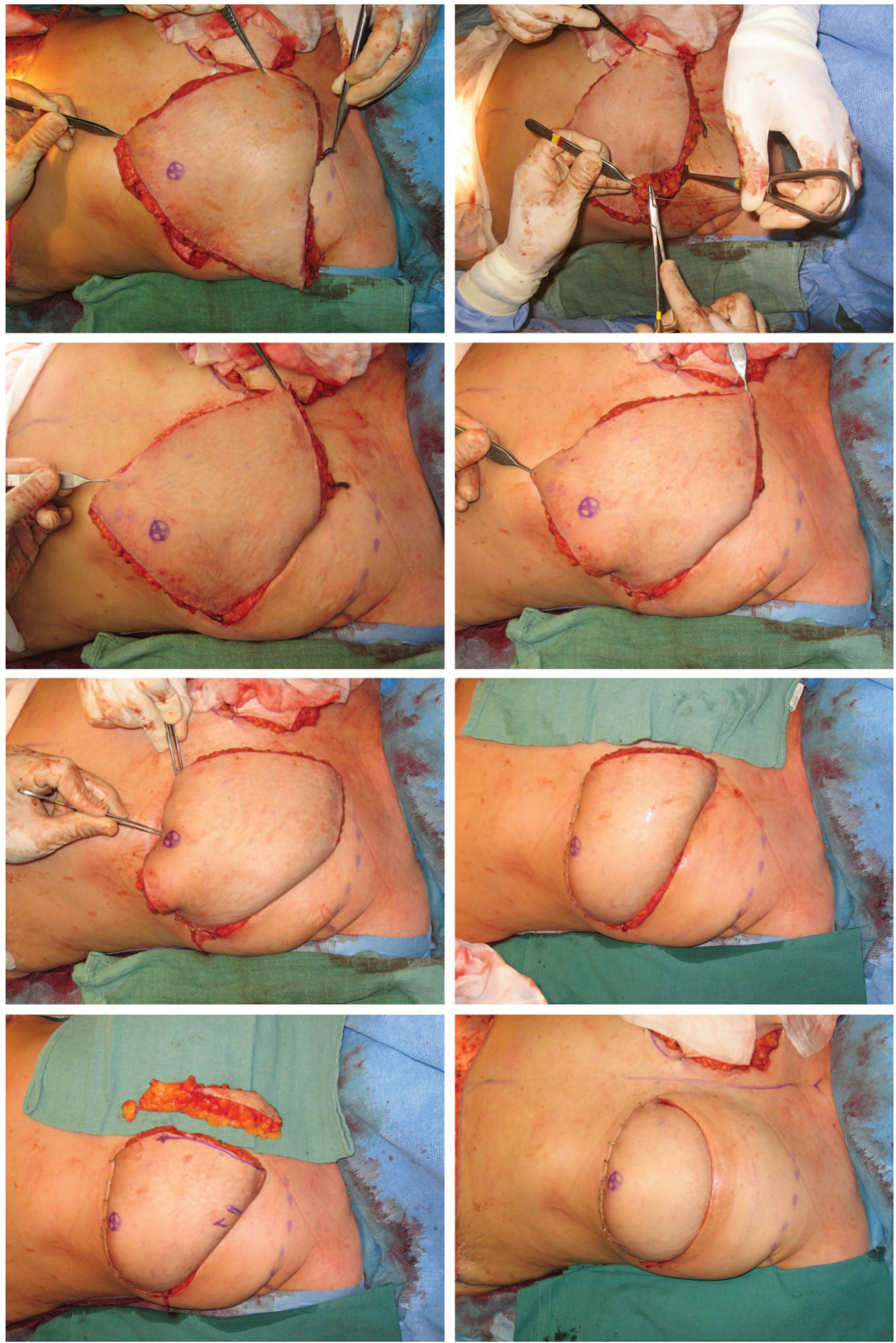

Fig. 4. Step-by-step shaping of a deep inferior epigastric artery perforator flap in a bilateral secondary autologous breast reconstruction. (Above, left) The flap is positioned over the left breast: the inferior border of the flap with the 
bilateral tattoos, even over the normal contralateral areola, provide the most natural results. Unfortunately, tattoos keep on fading at a slower pace and may need to be repeated several years later.

For autologous reconstructions, we like to follow the principles of classic sculpturing. A large block of material (in this instance, fat tissue) is placed on a platform, here obviously the chest wall. The first operation is intended to create a rough "basic" shape by removing larger pieces of fat from the newly transplanted flap. The flap appears like a breast but the volume and shape are not yet perfect after the first procedure. The total volume of the flap is left with a surplus of 10 to 15 percent to allow removal of smaller amounts of fat during the second procedure. Six months after transplantation, the flap has undergone the effects of gravity; has acquired a new, more natural shape; and will be at its final position on the footprint. To obtain perfect symmetry, it is wiser to remove small fat deposits and excess skin during the second procedure by relatively simple techniques such as direct excision and/or liposuction than it is to add more tissue with more involved techniques such as local flaps or lipofilling. The flap can easily be sculptured on the table with the patient in the sitting position until symmetry is perfect (Fig. 5).

It is our preference not to perform immediate autologous reconstructions when it is certain that postmastectomy irradiation will be performed. Temporary expanders are placed in these circumstances to preserve the mastectomy skin flaps, and delayed primary reconstruction is performed 6 months after finishing the last adjuvant therapy (i.e., chemotherapy or radiotherapy). If it is uncertain whether postoperative radiotherapy will be performed, we make the reconstructed breast 15 to 20 percent larger than the desired final volume.

\footnotetext{
Fig. 4. (Continued) superficial epigastric vein (microforceps) is placed cranially with the periumbilical tissues facing caudally. (Above, right and second row, left) Scarpa's fascia of the lateral corner of the flap is anchored to the pectoral fascia at the anterior axillary fold. (Second row, right and third row, left) The lateral fullness of the breast is determined: the more the flap is moved medially, the less bulging there is in the lower outer quadrant. Temporary skin staples are used to easily reposition the flap and facilitate shaping. (Third row, right and below, left) Turning the thickest periumbilical part of the flap onto itself will increase projection in the lower and central part of the flap. (Below, right) Removal of medial fat excess and deepithelialization of the upper part of the flap finalizes the shaping.
}

Postradiotherapy sclerosis is often responsible for a volume loss of approximately 10 percent, and we need an additional 10 percent surplus to preserve the liberty of removing fat to shape the breast.

We never perform a nipple reconstruction at the time of transplantation and delay nipple reconstruction until a later stage because of an unacceptably high rate of venous congestion. Most likely, there is a significant challenge to the blood flow in the dermal plexus just after microvascular transfer that makes the failure rate of such a "flapon-a-flap" significantly higher than performing a nipple reconstruction on a well-healed flap. Also, it is virtually impossible to determine the exact location of the new nipple on the breast mound, as the flap will shift an unpredictable distance on the chest wall in the postoperative phase. However, it remains important to plan ahead, and so we retain an excess of skin of approximately $6 \times$ $4 \mathrm{~cm}$ at the center of the breast mound to be elevated in later nipple reconstruction.

The shaping of the flap to form the conus is mostly performed by correct positioning by means of the three-key-suture technique as described above. In rare cases, the flap can be very thin, making it impossible to create sufficient projection in the lower part of the breast. The skin surface in such cases is too large, and too many wrinkles will develop in the skin paddle if one attempts to push the flap together. In that case, the flap can be folded onto itself over the midline with the skin on the outside. The tips of the flap are rounded by resecting the corners. The tips are placed not exactly over each other but next to each other. One tip is sutured below the pectoralis tendon; the other one, slightly more medial. By so doing, the upper pole is nicely filled. The rest of the shaping takes place as with half a flap in bilateral reconstructions. The perforator vessels lie in between both halves of the flap and exit medially toward the internal mammary artery and vein.

\section{IMPLANT RECONSTRUCTION}

\section{Footprint}

Regardless of timing or side of reconstruction, the footprint needs to be prepared in a way similar to that used with autologous reconstructions. There are nevertheless some specific differences. In primary skin-sparing mastectomies, the footprint and overlying skin are preserved and the footprint needs to be created or restored by undermining or resuturing as described earlier.

In secondary reconstructions, a new pocket with a new footprint needs to be created. After 

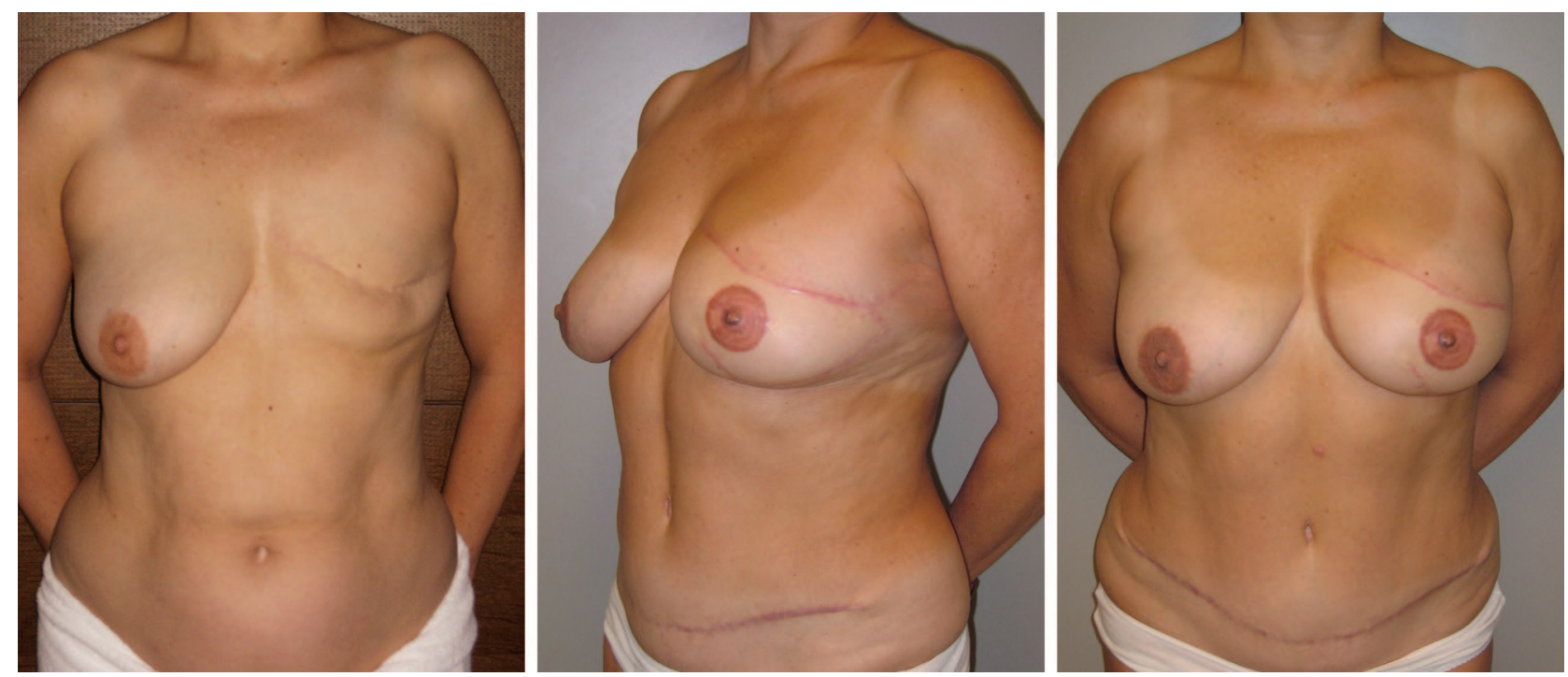

Fig.5. Preoperative (left) and postoperative views (center and right) of the shape of the breast after unilateral secondary autologous breast reconstruction by means of a deep inferior epigastric artery perforator flap and later nipple reconstruction and tattoo of the nipple-areola complex. This patient refused any contralateral breast surgery.

resecting the mastectomy scar, both mastectomy flaps are undermined up to the preoperative markings of the borders of the footprint. The contralateral breast can be very helpful for determining the exact location of these borders.

An important difference is that a second dissection below the pectoralis major muscle and eventually below the serratus anterior fascia needs to be performed to obtain muscle-fascia coverage. The extent of this undermining will depend on the dimensions of the implant but is never to exceed the borders of the footprint. It is often necessary to cut the lower origin of the pectoralis major muscle to lower the footprint down to its correct position. Depending on the dimensions of implant used, the footprint needs to be 1 to $2 \mathrm{~cm}$ wider than the implant to allow sufficient expansion of the pocket, but dissection must never surpass the outer borders of the normal breast.

\section{Conus}

The conus is made entirely of the implant itself. The shape of the implant determines the final shape of the breast. It is unclear to what extent the compression of the upper pole of a round implant by the overlying pectoralis muscle deforms the implant and thereby creates a natural slope with sufficient projection in the lower pole. Today, mostly anatomically shaped implants are used for complete breast reconstruction to ensure a natural shape.
Expanders, permanent expandable implants, and (cohesive) silicone gel- or salinefilled implants differ by only their volume and filling. The surgeon chooses the volume and dimensions (i.e., height, width, and projection) of the conus but cannot alter the shape of the implant. Fine tuning of the shape is impossible. The height and width of the implant are determined by the dimensions of the footprint and vice versa. In general, higher implants are used to fill up the infraclavicular part of the breast, in comparison with implants used for breast augmentation. Some companies produce specific implants with increased projection to be used in implant reconstruction. Until now, the utility of these expensive implants have not been proven. Sometimes, larger volume breast reconstruction can be performed only by placing implants that go over the limits of the footprint. This may result in an awkward appearing breast.

The main disadvantage of using an implant, next to the commonly known drawbacks of foreign bodies, is the possible displacement on the chest wall caused by pectoralis movements, exterior forces, and/or capsular contracture. This displacement will immediately deform the entire breast, resulting in an unnatural breast. Visible rippling of the implant and deformation of the implant by capsular contracture are other important problems that will cause distortion of the conus. 


\section{Envelope}

The skin envelope will cover the implant together with the pectoralis muscle. Draping of the skin proceeds in the same way as with autologous reconstructions. The lower skin flap is preserved and all scar tissue is removed from its inner surface. If there is a shortage of skin in secondary reconstructions or in cases where the mastectomy flaps cannot be adequately stretched, an expander needs to be placed first. Expansion continues until enough skin surface is obtained to fit the permanent implant. The fibrosis and sclerosis caused by radiotherapy will impede expansion of the skin or even lead to contraction of the skin and the subcutaneous layers. Lack of elasticity and contraction and deformation of the envelope will significantly deteriorate the final aesthetic result. For those reasons, many believe that implant reconstruction is incompatible with radiotherapy administered either before or after the placement of the implant. Implant reconstruction together with a pedicled latissimus dorsi or thoracodorsal artery perforator flap can be a valuable alternative to add more coverage of the implant and to insert more skin between the edges of the mastectomy flaps. If the skin envelope is too large, a simultaneous skin resection following the Wise pattern or other mastopexy design can be performed.
Phillip N. Blondeel, M.D., Ph.D.

Department of Plastic and Reconstructive Surgery University Hospital Ghent De Pintelaan 185, 2K12C B-9000 Ghent, Belgium phillip.blondeel@ugent.be

\section{REFERENCES}

1. Holmström H. The free abdominoplasty flap and its use in breast reconstruction: An experimental study and clinical case report. Scand J Plast Reconstr Surg. 1979;13:423-427.

2. Hartrampf CR, Scheflan M, Black PW. Breast reconstruction with a transverse abdominal island flap. Plast Reconstr Surg. 1982;69:216-225.

3. Bostwick J III, Vasconez LO, Jurkiewicz MJ. Breast reconstruction after a radical mastectomy. Plast Reconstr Surg. 1978;61:682-693.

4. Koshima I, Soeda S. Inferior epigastric artery skin flaps without rectus abdominis muscle. Br J Plast Surg. 1989;42:645-648.

5. Allen RJ, Treece P. Deep inferior epigastric perforator flap for breast reconstruction. Ann Plast Surg. 1994;32:32-38.

6. Blondeel P. One hundred free DIEP flap breast reconstructions: A personal experience. Br J Plast Surg. 1999;52:104-111.

7. Blondeel PN, Boeckx WD. Refinements in free flap breast reconstruction: The free bilateral deep inferior epigastric perforator flap anastomosed to the internal mammary artery. $\mathrm{Br}$ J Plast Surg. 1994;47:495-501.

8. Fujino T, Harasina T, Aoyagi F. Reconstruction for aplasia of the breast and pectoral region by microvascular transfer of a free flap from the buttock. Plast Reconstr Surg. 1975;56:178-181.

9. Allen RJ, Tucker C Jr. Superior gluteal artery perforator free flap for breast reconstruction. Plast Reconstr Surg. 1995;95: 1207-1212.

\section{American Society of Plastic Surgeons Mission Statement}

The mission of the American Society of Plastic Surgeons ${ }^{\circledR}$ is to support its members in their efforts to provide the highest quality patient care and maintain professional and ethical standards through education, research, and advocacy of socioeconomic and other professional activities. 